\title{
EL ESPACIO TURÍSTICO DE NIEVE EN ESPAÑA. PERSPECTIVA TERRITORIAL Y REVISIÓN TIPOLÓGICA
}

\section{The snow tourist areas in Spain. Territorial perspective and typological revision}

\author{
Antonio A. Ramón Ojeda ${ }^{1 *}$, Sarah López Rivero ${ }^{2}$ \\ ${ }^{1}$ Departamento de Geografía de la ULPGC. \\ ${ }^{2}$ Facultad de Empresariales, Economía y Turismo (ULPGC)
}

Identificador ORCID de los autores y e-mail Antonio A. Ramón Ojeda: http://orcid.org/0000-0002-4578-5185. Email: toni.ramon@ulpgc.es. Sarah López Rivero: https://orcid.org/0000-0001-7848-0874. Email: sarah.lopez101@alu.ulpgc.es *Autor de contacto.

Recibido: 24-04-2019. Aceptado: 14-06-2019. Fecha de publicación on-line: 31-07-2019

Citation / Cómo citar este artículo: Ramón Ojeda, A. A., López Rivero, S. (2019). El espacio turístico de nieve en España. Perspectiva territorial y revisión tipológica. Pirineos, 174, e046. https://doi.org/10.3989/pirineos.2019.174006

RESUMEN: Las instalaciones para la práctica de deportes de invierno en las montañas españolas han contribuido a producir espacios turísticos asociados a las estaciones de esquí. El presente estudio aborda, a partir de diferentes clasificaciones del espacio turístico de montaña asociado a la práctica de deportes de invierno, una propuesta de clasificación del espacio turístico de nieve en España. Para ello se ha elaborado un inventario de las 33 estaciones pertenecientes a ATUDEM que incluye 28 centros de modalidad alpina y 5 de esquí nórdico. En primer lugar, se ha realizado una revisión bibliográfica, con especial atención a los intentos de clasificación de estos espacios llevados a cabo en el ámbito de los Alpes y también en España. Seguidamente, a partir del inventario extenso de las estaciones españolas y tras elaborar esquemas territoriales de cada caso con el fin de estudiar las relaciones geográficas entre componentes espaciales, se propone una clasificación del espacio turístico de nieve y se describen sus características principales. Las cuatro tipologías posibles son: 1. Espacios complejos polinucleares y polivalentes. Estaciones de esquí asociadas a complejos turísticos. 2. Espacios simples mononucleares y especializados que conforman estaciones aisladas con urbanización turística. 3. Espacios simples mononucleares y especializados que conforman estación aislada más hotel o inmueble de apartamentos turísticos. 4. Espacios simples mononucleares y especializados que conforman estación aislada sin alojamientos en el ámbito inmediato.

PALABRAS CLAVE: Estación de esquí; espacio turístico de nieve; área de influencia; propuesta de clasificación.

ABSTRACT: The facilities for the practice of winter sports in the Spanish mountains have contributed to produce touristic spaces associated with ski resorts. The current study approaches, from the different mountain's classifications, touristic space combined with the practice of winter sports, a proposal of classification of the touristic snow area in Spain. For this purpose, an inventory of the 33 stations belonging to ATUDEM has been drawn up, which includes 28 alpine and 5 Nordic ski centers. First of all, a bibliographic review was carried out with special attention 
to the attempts to classify these spaces carried out in the area of the Alps and also in Spain. From the extensive inventory of Spanish stations and after developing territorial diagrams for each case in order to study the geographical relationships between spatial components, a classification of the snow tourism space is proposed, and its main characteristics are described. The four possible typologies are: 1. Multinuclear and polyvalent complex spaces. Ski resorts associated with tourist complexes, 2. Mononuclear and specialized single spaces that make up isolated ski resorts with tourist urbanization. 3. Mononuclear and specialized single spaces that make up isolated ski resort with hotel or tourist apartments and 4. Mononuclear and specialized single spaces that make up an isolated ski resort without accommodation in the immediate area.

KEY WORDS: Ski station; snow touristic space; influence area, classification design.

\section{Introducción}

El objeto del presente estudio es el espacio turístico de nieve asociado a la práctica del esquí en España. Se aborda éste desde una perspectiva territorial con el objetivo principal de realizar un análisis geográfico y establecer una clasificación general. Para ello, en primer lugar se lleva a cabo una revisión bibliográfica y discusión de la literatura que, desde un enfoque espacial, ha abordado los procesos de creación y desarrollo de destinos turísticos de esquí. Interesa especialmente comprender el sistema territorial que se encuentra asociado a este tipo de espacios turísticos. Con este fin se estudian: el tipo de recurso que se explota y sus características, las condiciones generales de las estaciones de esquí, el sistema de poblamiento especializado que se vincula con ellas, el origen y evolución de cada espacio y, finalmente, la estructura territorial que se ha generado como resultado de la implantación de las estaciones de esquí.

A partir de estudios similares elaborados por otros autores, que han tenido como principal referencia el ámbito alpino, se pretende comprobar su validez para el caso español. Para ello, y tras la revisión de la bibliografía existente, se elabora un inventario del espacio turístico asociado a la práctica del esquí en España ${ }^{1}$, se analiza cada caso con especial atención al proceso de producción y transformación espacial y se elabora finalmente una propuesta tipológica.

El espacio turístico de montaña español es utilizado principalmente por usuarios domésticos, pues el número de visitantes extranjeros $(8,9 \%)$ es muy poco significativo (Atudem, 2018; Vanat, 2018). En este sentido, BaqueiraBeret es la única estación donde la cifra de esquiadores foráneos superó los cien mil (158.000 en la temporada 2017/18 según Atudem). Actualmente, el parque de estaciones de esquí en España se compone de 33 espacios con desigual grado de desarrollo. Es un número escaso en comparación con otros destinos, tanto en Europa: Alemania (498 estaciones), Francia (325 estaciones), Italia (349 estaciones), Austria (254 estaciones) o Suiza (193 esta- ciones), como en otros continentes: EE.UU. (481 estaciones), Canadá (280 estaciones) y Japón (547) 2 . Esta circunstancia afecta de manera directa al estudio pues, de una parte simplifica el muestreo, pero por otra dificulta detectar pautas comunes con las que poder establecer una clasificación de los 33 espacios considerados.

\section{2. Área de estudio. Síntesis del proceso de formación del espacio turístico de nieve en España}

¿Cómo ha sido la producción del espacio turístico de nieve en España? Comprender este proceso puede dar algunas claves para interpretar los destinos de esquí y sus tipologías. No ha sido posible estudiar dicho proceso de forma pormenorizada para cada caso, pero se expone seguidamente un esquema general del mismo por entender que resulta esencial como paso previo a la clasificación de este tipo de espacios.

La práctica del esquí se extiende de manera tardía por los sistemas montañosos españoles (Cardona \& Dupre, 1985), a finales del siglo XIX y principios del XX. Al comenzar la década de 1950 sólo existían cinco estaciones de esquí: La Molina y Val de Núria (1943) en el Pirineo catalán, Navacerrada (1954) en Guadarrama, Candanchú (1945) en el Pirineo aragonés y Pajares (1954) en la Cordillera Cantábrica. Cada una de ellas disponía de una buena conexión ferroviaria, lo que sin duda resultó clave en sus desarrollos tempranos (Fernández et al., 1990).

La transformación se produjo en la década de 1960 (Martos, 2002; Laguna \& Nogués, 2002; Lasanta et al., 2014) y década de los setenta (López Palomeque, 1996), en especial tras la aprobación por parte del Gobierno de una línea de crédito y subvenciones en octubre de 1965. Al amparo de la Ley de Centros y Zonas de Interés Turístico (1963) el Ministerio de Turismo había asumido las competencias para aprobar planeamiento, ocupaciones de suelo, expropiaciones, créditos hipotecarios, ayudas y subvenciones (Fernández et al., 1990) y bajo su amparo se construyeron 28 nuevas estaciones invernales. El pro-

\footnotetext{
${ }^{1}$ Para el presente trabajo se ha utilizado el censo de estaciones de esquí de la Asociación Turística de Estaciones de Esquí y Montaña (ATUDEM). Recuperado de http://www.atudem.es/.

2 Vanat, L. (2018). https://vanat.ch/international-report-on-snow-mountain-tourism.shtml [consultada 1/12/2018]. Se contabilizan las áreas de esquí con 5 remontes o más.
} 
ceso se basó en promociones turísticas que tenían como principal objetivo aumentar la capacidad receptiva, siguiendo las estrategias promovidas por el Ministerio de Información y Turismo y que también se emplearon en el Mediterráneo y Baleares (OIU, 1973).

Muchos de los nuevos centros de esquí, por ejemplo La Pinilla ${ }^{3}$ o Astún ${ }^{4}$, se construyeron tras previa tramitación de expediente de declaración como Centro de Interés Turístico por parte de sus promotores (Galiana \& Barrado, 2006) y se levantaron sin ningún género de articulación con todo lo que le rodea, como unidades autónomas desarticuladas del paisaje (OIU, 1973: 12).

En estos años de desarrollismo, el modelo de estación integral francesa era el favorito, pero se encontraba lejos de las posibilidades españolas (Fernández et al., 1990), sobre todo a nivel de inversores. No obstante, el nuevo paradigma tuvo éxito y supuso la construcción de las estaciones directamente en los campos de nieve, junto a los dominios esquiables, hecho que explica el predominio de las estaciones aisladas. Esta circunstancia supuso la construcción ex profeso de infraestructuras de transporte que comunicaran los nuevos enclaves con el sistema general de carreteras o ferroviario, un aspecto fundamental en la tipología resultante ( 28 de los 33 casos considerados). La rentabilidad de estos nuevos centros quedó vinculada a los beneficios inmobiliarios más que a la explotación de las propias estaciones (López Palomeque, 1996), dando lugar a expansiones urbanísticas que carecieron de una planificación adecuada. El planeamiento de las estaciones de invierno del Pirineo no ha considerado suficientemente la importancia de las características especificas de su emplazamiento, (OIU, 1973: 13), afirmación de absoluta vigencia en la actualidad y aplicable a todos los enclaves españoles de turismo de esquí construidos durante esa época.

Tras el boom constructivo de los sesenta y setenta, los años ochenta representaron una crisis para el sector y la entrada de capital público como fórmula de reactivación. Esta circunstancia se generalizó para el conjunto de las estaciones españolas (Porras, 2005), resultando muy común. Hay muchos ejemplos: Astún, Espot, Formigal, La Molina etc. A finales de esta década se introducen cañones de nieve en las estaciones, permitiendo prolongar la temporada y, con ello, la rentabilidad (Bayón, 1999). Se imprime un nuevo impulso y aires de modernización, mientras el sector público se afianza en los espacios turísticos dedicados al esquí a través de fuertes inversiones (López Palomeque, 1996).

A finales de los noventa y principios de 2000 surge un nuevo tipo de estación invernal, especializada ahora en el esquí nórdico (Llanos del Hospital, Larra-Belagua, Puerto de la Ragua y Lles, que se reinventa tras un fracasado intento en la modalidad alpina). Estos nuevos centros compatibilizan diferentes actividades deportivas como montañismo, senderismo, rafting y esquí de fondo, y producen un espacio más sostenible en términos ambientales, pues no precisa de las infraestructuras propias del esquí alpino.

Desde finales de siglo se ha observado una reconversión de muchas de las viejas instalaciones, lo que parece una tendencia entre los gestores de estos espacios (Bourdeau, 2009). El número de remontes se ha ido reduciendo

Tabla 1: Síntesis del proceso de producción del espacio turístico español de nieve. Fuente: elaboración propia.

Table 1: Summary of the production process of the Spanish snow tourism areas. Source: Own elaboration.

\begin{tabular}{|l|l|l|}
\hline \multicolumn{1}{|c|}{ Etapa } & \multicolumn{1}{|c|}{ Periodo } & \multicolumn{1}{c|}{ Proceso } \\
\hline Exploración & $\begin{array}{l}\text { Finales del s. XIX y } \\
\text { principios del XX }\end{array}$ & $\begin{array}{l}\text { Difusión del esquí. Primeros aficionados hacen uso de las zonas de nieve } \\
\text { próximas a pueblos y puertos de montaña. }\end{array}$ \\
\hline Descubrimiento & Años 20-30 & $\begin{array}{l}\text { Incremento de la demanda. Mejora de las conexiones ferroviarias. Se } \\
\text { construyen los primeros refugios y clubes de alpinismo. }\end{array}$ \\
\hline Redescubrimiento & Años 40-50 & $\begin{array}{l}\text { Tras la posguerra, se reactiva la demanda del turismo de montaña. Mejora } \\
\text { paulatina de la red viaria y construcción de nuevos refugios de montaña. } \\
\text { Primeros remontes mecánicos como resultado de iniciativas privadas. }\end{array}$ \\
\hline Producción & Años 60-70 & $\begin{array}{l}\text { Construcción de la mayoría de las estaciones modernas. Predominio de } \\
\text { iniciativas privadas con apoyo de los agentes públicos. Escasa planificación } \\
\text { integral resultado de lo cual predominaron las estaciones aisladas. }\end{array}$ \\
\hline Consolidación & Años 80-90 & $\begin{array}{l}\text { Intervención de la administración pública mediante subvenciones, incentivos, } \\
\text { e incluso la gestión directa. }\end{array}$ \\
\hline Renovación & Siglo XXI & $\begin{array}{l}\text { Continúa la participación pública. Modernización y diversificación de las } \\
\text { instalaciones. Espacios polivalentes cada vez más complejos. Asociación de } \\
\text { dominios y gestión compartida. }\end{array}$ \\
\hline
\end{tabular}

\footnotetext{
${ }_{3}^{3}$ Decreto 3165/1971, de 25 de noviembre de 1971 (BOE 311 de 29/12/1971).

${ }^{4}$ Decreto 2229 de 24 de junio de 1975 (BOE 25 de septiembre de 1975).
} 
mediante una reorganización y mejora tecnológica y de capacidad de los mismos, lo que a su vez ha permitido reducir el impacto global de las estaciones de esquí.

El proceso de producción del espacio turístico español se puede resumir en seis etapas (Tabla 1), de las cuales, las tres primeras son de exploración y descubrimiento y las tres últimas de verdadera creación de nuevos espacios.

En definitiva, el desigual proceso de producción del espacio turístico de nieve español se debe a diversas circunstancias de las cuales, las principales son: la desigual participación inversora de los agentes, tanto públicos como privados, las condiciones geográficas, la estructura territorial y el grado de desarrollo de cada caso de estudio.

\section{Revisión bibliográfica y estado de la cuestión}

Aunque el presente estudio no pretende ser una revisión bibliográfica, sí que ha resultado imprescindible llevar a cabo una discusión de los modelos más relevantes que se han elaborado para explicar los desarrollos turísticos de las estaciones de esquí. Para ello, se ha seguido el método de la declaración PRISMA a la hora de seleccionar la bibliografía ${ }^{5}$. A este respecto, en algún caso no ha sido posible acudir a las fuentes originales, recurriendo entonces a fuentes secundarias. Los modelos considerados pueden ser analizados desde diferentes enfoques o perspectivas, no todas ellas geográficas, y se organizan seguidamente según orden de aparición.

Cronológicamente, la primera clasificación del desarrollo de campos de esquí (Barrado \& Calabuig, 2001: 131) la propone Pierre Préau en 1968 teniendo como ámbito de estudio los Alpes septentrionales (Préau, 1968). Dos aspectos destacamos de su estudio: una clasificación en cuatro grandes grupos de categorías de estaciones: Grandes complejos turísticos desarrollados en el marco de grandes autoridades locales; estaciones de importancia media ancladas en pueblos grandes; estaciones creadas desde cero y centros secundarios. Y una conclusión: la dificultad de establecer una clasificación, que en palabras del autor, roza lo posible (Préau, 1968: 138).

Otra clasificación, ésta de marcado cariz geográfico, es la propuesta por Defert en 1972, quien clasifica las estaciones según su emplazamiento, distinguiendo entre estaciones de valle, estaciones puerto, estaciones de balcón o terraza y estaciones de circo terminal (Defert, en Porras, 2005: 154). Se trata de una clasificación muy simple en sus planteamientos, pues tan solo tiene en cuenta la posición topográfica que ocupan los espacios de nieve.

Con posterioridad, Barker (1982) realiza una revisión histórica del cambio de paradigma y de los diferentes modelos de desarrollo del espacio turístico que han tenido lugar en los Alpes, especialmente a partir de la segunda mitad del siglo XX, ya que es el período que coincide con la masificación de esta modalidad turística. Aunque no tiene por objeto elaborar una clasificación, sí que acaba por hacerlo cuando observa diferencias entre los Alpes Occidentales y los Alpes Orientales. Mientras que los primeros parecen haber optado por un desarrollo de estaciones integrales (grandes resort turísticos aislados y autónomos que se sitúan a una mayor altitud, en torno a los $2.000 \mathrm{~m}$ ), en los Alpes Orientales se opta por un modelo que asegura una mayor integración y simbiosis entre turismo y agricultura tradicional de montaña (Barker, 1982: 415).

Este aspecto sería más tarde retomado por LozatoGiotart (1990) quien, apoyado en los estudios que le precedieron, elabora una propuesta que establece cuatro categorías, basándose principalmente en criterios geográficos y cronológicos. Sus tipologías se resumen en espacios polinucleares y multipolares, según el número de núcleos de población y focos de atracción que existan, así como su grado de especialización. Retoma conceptos como el empleado por Barker (1982) en referencia a las estaciones integrales; directamente denomina modelo tirolés al tipo de desarrollo que dicha autora había descrito en los Alpes Orientales al referirse a los espacios de nieve territorialmente más integrados. La importancia del modelo de Lozato-Giotart (1990) es que propone una clasificación a partir del análisis del entorno próximo a las estaciones de esquí y las relaciones espaciales que se establecen entre los recursos naturales, las infraestructuras (turísticas y de transporte) y la población local. Este hecho resulta especialmente relevante para el presente estudio.

Lozato-Giotart (1990) elabora una propuesta con cuatro tipos de estaciones que ordena cronológicamente, distinguiendo entre estaciones de primera generación (hasta 1940), segunda generación (años 40 y 50), tercera generación (años 60 y 70) y cuarta generación (a partir de los 80). Las primeras son espacios vinculados a pueblos tradicionales de montaña que han derivado hacia destinos turísticos de nieve; las segundas corresponden a la primera fase de industrialización de la nieve y la construcción de nuevas estaciones situadas a mayor altitud, pero aún con cierta vinculación con el sistema tradicional de poblamiento; las terceras son verdaderas "fábricas de esquí", macro-estaciones integrales aisladas que se emplazan hasta 2.000 metros de altitud y carecen de relación territorial con los núcleos tradicionales; la cuarta es una reacción "ambientalista" al impacto generado durante años y vuelve al modelo tirolés, dotando los espacios de nieve de una nueva dimensión más integrada en el territorio y el paisaje.

Sobre un estudio en Nueva Zelanda, Pearce (1989, 1999), elabora su modelo basándose en una clasificación que considera más la participación de los agentes que intervienen en el proceso de producción espacial de las es-

\footnotetext{
5 La búsqueda inicial ofreció 647.878 resultados, quedando restringido tras los primeros filtros a 2.706 referencias de las que finalmente se revisaron 43. Sólo las empleadas en el presente trabajo figuran recogidas en la bibliografía. http://www.prisma-statement.org/.
} 
taciones que el resultado territorial en sí mismo. Sin embargo, no estudia la producción de los nuevos espacios, ni cómo quedan estos configurados.

Mesplier y Bloc-Duraffour (2000) aportan una síntesis de clasificaciones realizadas hasta ese momento, aunque con preeminencia de la propuesta de Lozato-Giotart (1990). No se trata en sí mismo de un nuevo estudio específico, sino únicamente de una revisión comentada y actualizada. No aporta novedades a lo realizado, sólo una nueva sistematización en la que siguen manteniendo 4 tipos de estaciones de altitud (Mesplier \& Bloc-Duraffour, 2000: 131 y ss.), que se diferencian por su tamaño, emplazamiento, altitud, arquitectura y nivel de equipamiento.

En lo referente a España, hay que destacar la escasez de estudios, muchos de los cuales se redactaron en los años 70, justo el momento del gran boom constructivo de los centros de esquí. Este es el caso de Cirici (1973), quien cita dos tipos de espacios de esquí: parques y estaciones. Los primeros surgen en zonas próximas a núcleos de población y se justifican por su fácil accesibilidad, mientras que las estaciones están pensadas para usuarios que se desplazan distancias más largas y, por tanto, demandan infraestructuras de alojamiento, resultado de lo cual son espacios más complejos. Sin embargo, el propio autor seguidamente advierte que los verdaderos centros de esquí se encuentran en los Alpes, Las Rocosas, Japón y Nueva Zelanda.

Una propuesta interesante es elaborada también en 1973, en este caso por la Oficina de Información Urbanística $^{6}$ (OIU, 1973) catalana. En ella se mencionan tres tipos de estaciones de esquí clasificadas según la duración de la estancia de los usuarios y la distancia respecto a Barcelona. Esta ciudad es considerada como mercado principal de las estaciones pirenaicas, espacio sobre el que se elabora el estudio. El primer grupo de estaciones estaría formado por aquellas que están localizadas a menos de $150 \mathrm{~km}$ de la Ciudad Condal. Son espacios polivalentes donde se citan tanto deportistas como senderistas y otros excursionistas. Constituyen destinos de fin de semana con escasa oferta hotelera. Frente a esta tipología nos encontramos con otras dos que se localizan a más de 250 $\mathrm{km}$ de Barcelona, o incluso a más de $300 \mathrm{~km}$. En ambos casos son estaciones deportivas, entendiéndose como tales espacios más especializados en los deportes de invierno. Además, su lejanía (respecto a Barcelona) conlleva necesariamente estancias más largas (de 3 o 4 días, según los autores) y por tanto la producción de un destino dotado de infraestructuras de alojamiento. Para diferenciar las tres tipologías establecen un índice que relaciona la capacidad de las estaciones (esquiadores/hora) y la oferta de alojamientos. Este índice resulta más elevado cuanto más alejadas se encuentren las estaciones, llegando incluso a ser negativo en la primera tipología, y próximo a cero o positivo en la segunda y la tercera.

Con la popularización del esquí y la construcción de las primeras estaciones, desde el Ministerio de Informa- ción y Turismo se propuso la creación de "burgos turísticos" destinados a una clientela con menor poder adquisitivo (Fernández et al., 1990). La iniciativa no se concretó y no se desarrolló ningún destino de este tipo, aunque hubo estaciones, como por ejemplo Espot-esquí, que arrancó con la idea inicial de crear uno de estos espacios (López Palomeque, 1996). Esta tipología no tuvo desarrollo.

Ganyet (1985, en Porras, 2005) también elabora una propuesta que clasifica las estaciones pirenaicas según la distancia a los mercados principales. Este factor determinaría el uso de los espacios de nieve y la mayor o menor necesidad de construir alojamientos. Ganyet cita tres tipos: estación de permanencia (las más alejadas y con mayor infraestructura de alojamientos), estadio de nieve y parque de nieve (estos dos más próximos a centros urbanos, son espacios más polivalentes en cuanto a su uso y con menor desarrollo hotelero, en especial los parques, que son más pequeños).

Sobre esta base, López Palomeque (1996) propone una clasificación para las estaciones potenciales del Pirineo catalán según el tamaño y la funcionalidad, distinguiendo 3 tipos: Estancia (dominio grande y de calidad); familiar (menor dimensión y calidad) y parque de nieve (fácil acceso y equipamiento, con influencia local o regional). Constata, como lo hiciera previamente la OIU (1993), la diferencia entre los espacios más próximos a Barcelona, a los que se puede acudir en una sola jornada (Port del Comte, Rasos de Peguera, Masella, La Molina y Vallter 2000), y aquellos otros más alejados y que precisan de una estancia más prolongada (cita las comarcas de Val d'Aran, Alta Ribagorça y Pallars Sobirà). López Palomeque (1996: 29) indica que: Desde la perspectiva de su implantación espacial las estaciones de esqui se pueden agrupar en relación al modelo de su concepción y organización empresarial; al modelo de asentamiento o emplazamiento y al modelo territorial (...). Sin embargo, no lleva a cabo su clasificación según dichos criterios, añadiendo seguidamente que: “...sin duda, la modelización de estaciones de esqui que presenta más atractivo es la que se establece a partir del análisis del proceso de formación y la evolución de sus características en relación con su entorno inmediato, considerando tanto sus aspectos formales como funcionales. Nos referimos a la distinción de las estaciones por generaciones: primera, segunda, tercera y cuarta generación". Por lo que finalmente recurre a la clasificación de Lozato-Giotart (1990) como modelo de tipificación, advirtiendo que "la mayor parte de las estaciones catalanas encajan dentro de la segunda generación" y algunas presentan características "inherentes a la tercera generación: La Masella, Baqueira-Beret, Boi-Taüll o Portainé” (op. cit: 29).

Porras (2005) defiende su tesis doctoral sobre las estaciones de esquí españolas, pero lejos de realizar una tipificación, simplemente las ordena cronológicamente (estaciones construidas entre 1940 y 1960; 1960 y 1980 y

\footnotetext{
${ }^{6}$ De Barcelona.
} 
posteriores a 1980). Por lo tanto, recurre al modelo de Lozato-Giotart (1990), sin que la suya se pueda considerar una propuesta de clasificación en sí misma. Por otra parte, hay que advertir que tipificar el espacio turístico de nieve no es en absoluto uno de los objetivos de Porras (2005), sino una cuestión tangencial en su estudio.

Otro trabajo (Tritu, 2018), este muy reciente y no publicado en ningún medio académico ${ }^{7}$, lleva a cabo una clasificación de las estaciones de esquí españolas según su "momento de potencia" (MP), un índice definido por el Service Technique des Remontées Mécaniques et des Transports Guidés y adaptado para el caso español. Dicho índice mide la capacidad de los remontes para hacer ganar altitud a los usuarios, o expresado de otra manera: la capacidad de las estaciones de esquí para transportar pasajeros. El organismo francés estipuló en 2014 los siguientes intervalos y clases:

- Estaciones Pequeñas: Inferior a $2.500 \mathrm{Km}$-Esqs/h de MP acumulado.

- Estaciones Medianas: 2.500-6.000 Km-Esqs/h de MP acumulado.

- Estaciones Grandes: 6.000-15.000 Km-Esqs/h de MP acumulado.

- Estaciones Muy Grandes: superior a 15.000 KmEsqs/h de MP acumulado.

Esta clasificación valora la capacidad de carga de las estaciones de esquí, pero no tiene en cuenta otros muchos aspectos geográficos, ni su origen, desarrollo, funcionalidad, especialización y estructura territorial. No obstante, es una propuesta objetiva a través de la cual se logra expresar la magnitud o dimensiones de estos espacios de una forma comparada.

En definitiva, los estudios sobre clasificación de las estaciones de esquí españolas son escasos y se centran principalmente en los Pirineos. No hemos encontrado estudios sistemáticos que aborden la totalidad del espacio turístico de nieve de España, ni propuestas tipológicas elaboradas ad hoc. Los trabajos posteriores a 1990 (López Palomeque, 1996; Porras, 2005) llevan a cabo un intento de sistematización a partir del modelo de LozatoGiotart (1990). Exponen una clasificación de las estaciones de esquí a partir de su ordenamiento cronológico, aspecto que se ha tenido en cuenta en el presente estudio con el fin de valorar su idoneidad.

\section{Metodología}

El presente trabajo tiene como punto de partida la revisión bibliográfica y discusión de las propuestas tipoló- gicas del espacio turístico de montaña vinculado a la práctica del esquí, con especial atención a aquellos estudios centrados en analizar la producción de dichos destinos desde una perspectiva territorial.

Para abordar el análisis geográfico del espacio de esquí se ha elaborado un inventario de 33 estaciones, las que forman parte de Atudem. De cada una se han considerado los datos que aparecen indicados en la tabla 2 .

Una vez consignadas las variables de estudio se elaboró una ficha de inventario para su posterior análisis, así como un banco de datos alfanuméricos y gráficos (Figura 1) referidos a las estructuras y condiciones territoriales particulares de cada caso. Esta base de datos tiene por finalidad poder estudiar las relaciones espaciales que se establecen a partir de la creación de la estación respecto de los recursos territoriales turísticos, las infraestructuras turísticas, la red viaria y el sistema de poblamiento. El estudio se lleva a cabo considerando dos ámbitos territoriales de análisis: el ámbito inmediato (consignado entre 0 y 5 $\mathrm{km}$ en torno al recurso territorial que constituye el principal atractivo de la actividad, esto es, la estación de esquí y sus dominios esquiables), y el ámbito próximo (entre 5 y $15 \mathrm{~km}^{8}$ ). Para determinar estas coronas de análisis se ha ensayado previamente con varios casos de estudio, lo que nos ha permitido fijar el diámetro territorial en torno a la estación. Se ha descartado un análisis del ámbito extenso por entender que escapa de los objetivos del presente estudio. No obstante, y con la única finalidad de poder determinar con mayor precisión el ámbito próximo de afección, sí que se han considerado las rutas viarias desde los principales ámbitos de procedencia de usuarios, esto es: Madrid, Barcelona, País Vasco (en especial Bilbao), Zaragoza, Málaga, Granada y Valencia.

Es preciso advertir que no ha sido posible establecer un área de influencia territorial debidamente justificada. En este sentido, Atudem contemplaba en sus estadísticas de 1998 una variable que denominan como "alojamientos en el área de influencia", para el que utilizaban un diámetro de $35 \mathrm{~km}$ a la redonda. La distancia ha desaparecido en las estadísticas oficiales actuales (Atudem, 2018), si bien siguen aportando un dato de alojamientos en el área de influencia, aunque sin precisar ya qué superficie cubre dicha área. Sobre este mismo aspecto, Lasanta, et al. (2007: 160-161) — desconocemos si atendiendo a datos de la propia Atudem-, hacen referencia a alojamiento "en los alrededores" de las estaciones, para posteriormente incluir en su ámbito de estudio aquellos municipios distantes hasta $100 \mathrm{~km}$. En otro trabajo (Lasanta, 2010) el propio Lasanta repite la misma variable, pero de nuevo no se consigna un diámetro preciso. Lasanta et al. (2014) en un interesante trabajo sobre Valdezcaray, mencionan un radio de $50 \mathrm{~km}$ (una

\footnotetext{
7 Tritu (20 de diciembre de 2018) Re: Clasificación de las Estaciones de Esquí Españolas y Portuguesa por Momento de Potencia. Temporada 2018/19. [informe en un foro en línea]. Recuperado de https:/www.nevasport.com/rmeee/art/36267/Clasificacion-de-las-Estacionesde-Esqui-Espanolas-y-Portuguesa-por-Momento-de-Potencia-Temporada-201819/ [consultada el 28 de diciembre de 2018].

${ }^{8}$ La media respecto al pueblo con ayuntamiento más cercano a las pistas se sitúa en $14,5 \mathrm{~km}$. En un radio máximo de $30 \mathrm{~km}$ estaría la totalidad de las estaciones.
} 
Tabla 2: Variables consignadas en la base de datos de las estaciones de esquí. Elaboración propia.

Table 2: Variables of the database of ski resorts. Source: Own elaboration.

\begin{tabular}{|c|c|c|c|}
\hline Variable & Unidad & Año/periodo & Opciones posibles \\
\hline Nombre de la estación & - & - & - \\
\hline Sistema montañoso & - & - & - \\
\hline Provincia & - & - & - \\
\hline Año de apertura & - & - & - \\
\hline Número de pistas & - & 2018 & - \\
\hline Kilómetros esquiables & $\mathrm{km}$ & 2018 & - \\
\hline Kilómetros nieve artificial & $\mathrm{km}$ & 2018 & - \\
\hline Remontes & Total tipos & 2018 & - \\
\hline Altitud & m.s.n.m. & - & - \\
\hline Cota máxima & m.s.n.m. & - & - \\
\hline Cota mínima & m.s.n.m. & - & - \\
\hline Cañones & $\mathrm{N}^{\mathrm{o}}$ de cañones & 2018 & - \\
\hline $\mathrm{N}^{\mathrm{o}}$ de balsas & $\mathrm{N}^{0}$ de balsas & 2018 & - \\
\hline Snowpark & $\mathrm{N}^{\mathrm{o}}$ de snowparks & 2018 & - \\
\hline Áreas de aparcamiento-remonte & $\mathrm{N}^{\mathrm{o}}$ de áreas & 2018 & - \\
\hline Superficie de aparcamiento & $\mathrm{m}^{2}$ & 2018 & - \\
\hline Capacidad & personas/hora & 2018 & - \\
\hline Momento de potencia acumulado (MPA) & $\mathrm{km} / \mathrm{esquiadores} / \mathrm{h}$ & 2018 & \\
\hline Clase según MPA & - & 2018 & $\begin{array}{l}\text { muy grande, grande, mediana y } \\
\text { pequeña }\end{array}$ \\
\hline Forfait vendidos en la temporada $2017-18$ & $\mathrm{~N}^{\mathrm{o}}$ de forfaits & $2017-18$ & - \\
\hline Días abiertos (media temporadas 2009-2018) & Días & $2009-2018$ & - \\
\hline Urbanización asociada & - & 2018 & si/no \\
\hline Hotel & - & 2018 & si/no \\
\hline Viviendas censadas & - & 2018 & - \\
\hline$\%$ Viviendas no principales & - & 2018 & - \\
\hline Alojamientos en la estación (plazas) 1998 & plazas & 1998 & - \\
\hline Alojamientos en la estación (plazas) 2018 & plazas & 2018 & - \\
\hline Alojamientos en 35 km8 (plazas) 1998 & plazas & 1998 & - \\
\hline Alojamientos en 35 km (plazas) 2018 & plazas & 2018 & - \\
\hline Viario de acceso & - & 2018 & $\begin{array}{l}\text { vinculación a puerto de montaña, } \\
\text { fin de vía, ramal específico }\end{array}$ \\
\hline Distancias a núcleo con ayuntamiento más cercano & $\mathrm{km}$ & 2018 & - \\
\hline Pueblo más cercano y urbanización si la hubiera & $\mathrm{km}$ & 2018 & - \\
\hline Propiedad & - & 2018 & Pública/privada \\
\hline Gestión & - & 2018 & Pública/privada \\
\hline
\end{tabular}

hora en coche) como margen para establecer el área de influencia de esta estación.

Por otra parte, la variable censal o administrativa (uso de estadísticas municipales o comarcales), aunque de uso frecuente (Perlik, 2011; Lasanta et al., 2013), no se demuestra operativa. Esto es así porque son muchos los ca- sos en los que los pueblos de los alrededores de las estaciones no forman parte del mismo municipio que esta última. El ejemplo más singular es el de Astún, que pertenece al municipio de Jaca, del que constituye un enclave situado a $33 \mathrm{~km}$ de la cabecera municipal. En este sentido, resulta de interés el trabajo de Lasanta et al. (2013), 


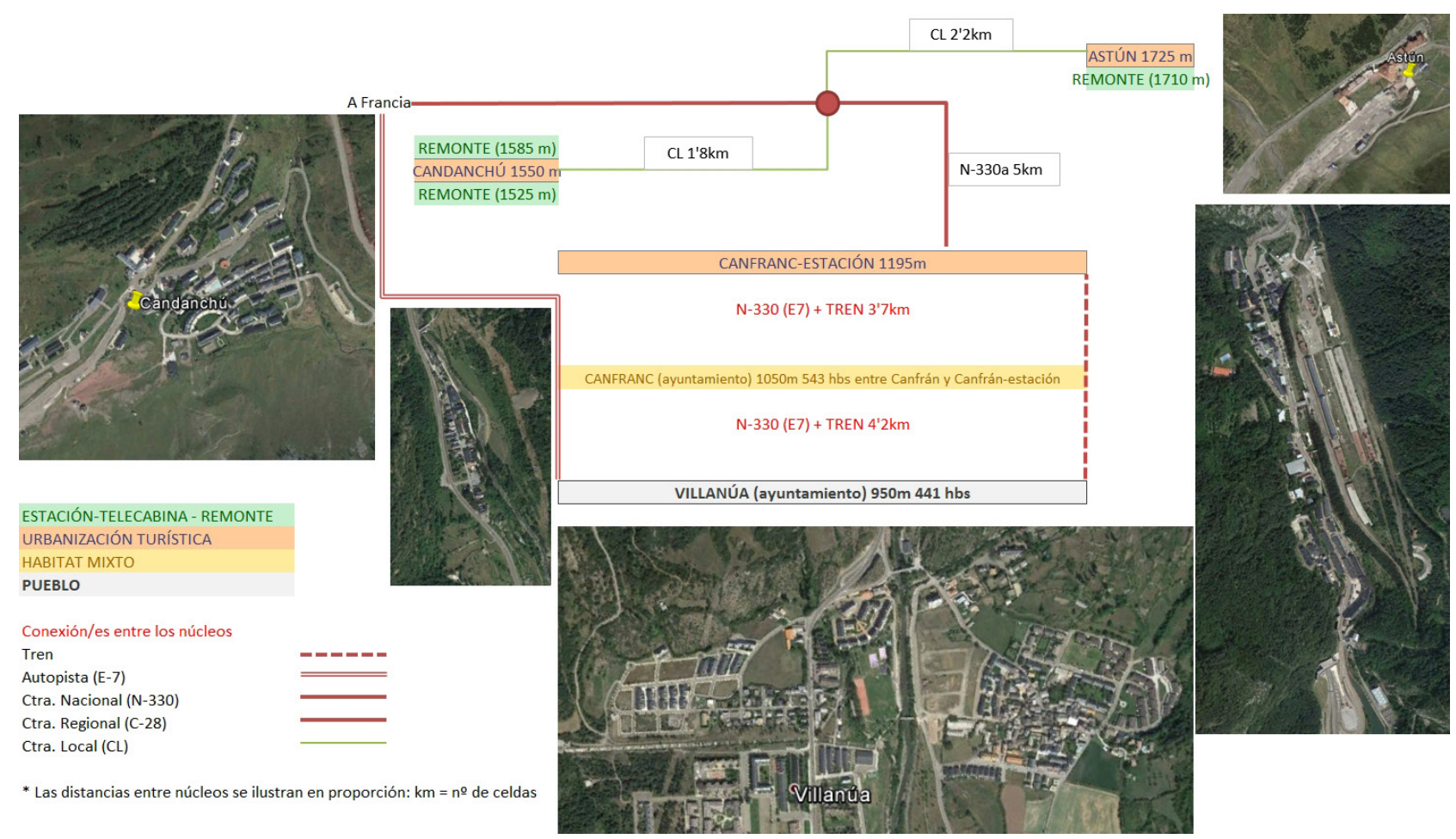

Figura 1 Ejemplo de croquis que expresa las relaciones territoriales (Caso de Candanchú-Astún). Fuente: elaboración propia. Figure 1: An instance of sketch that expresses territorial relationships (Candanchú-Astún). Source: Own elaboration.

pues correlaciona justamente la división administrativa municipal, la existencia de estación de esquí y el desarrollo territorial de los municipios cercanos. Este estudio establece una clasificación de los municipios según posean o no estación de esquí.

En definitiva, a este respecto debe aclararse que para el presente estudio no ha sido posible determinar un área sobre el que cada estación ejerza una "influencia” económica, territorial o demográfica. Por esta razón, se ha preferido hablar de ámbitos territoriales inmediato (0-5 km) y próximo $(5-15 \mathrm{~km})$, este último establecido de manera estimada a partir de las distancias que separan cada estación del sistema tradicional de poblamiento (núcleos habitados y entidades de población con ayuntamiento). Hay que matizar que el objetivo de estudio del presente trabajo es principalmente territorial y que establecer las coronas o ámbitos circundantes resulta suficiente, pero de cara a futuras investigaciones sería deseable ahondar sobre métodos de cálculo del área de influencia de los espacios turísticos de esquí.

Sobre los ámbitos inmediato y próximo se ha realizado un análisis de distribución espacial de los principales componentes del proceso de producción del espacio turístico en cuestión, es decir, los ya antes mencionados: recursos naturales, infraestructuras turísticas, de alojamiento y transporte (se obvian las promocionales), y sistemas de poblamiento y transportes. El análisis comprende las estructuras propias de las estaciones (edificaciones, remontes, cañones de nieve, balsas, áreas de aparcamiento, infraestructuras de alojamiento y viaria, etc.), variables geográficas como emplazamiento, orientación, altitud, cotas mínima y máxima, etc.; también se estudia el sistema de poblamiento (población, viviendas, alojamientos, segundas residencias, evolución urbanística, configuración del sistema, rutas principales de acceso, etc.).

A partir del análisis de componentes y tras considerar la idoneidad para el caso español de otros modelos, en especial el de Lozato-Giotart (1990), se establece una clasificación de las estaciones de esquí y sus características. Se concluye con una presentación de resultados en forma de propuesta tipológica y se aporta, por último, la bibliografía consultada.

\section{Discusión de las clasificaciones revisadas y nueva propuesta de clasificación de los espacios de esquí}

Ya con antelación se puso de manifiesto que muchas clasificaciones adoptan como criterio básico el momento de creación de las estaciones de esquí y por consiguiente su antigüedad. De hecho, los estudios más recientes sobre tipificación de las estaciones de esquí españolas no acaban de realizar una propuesta global (López Palomeque, 1996), o si lo hacen se limitan a proponer una clasificación según el año de creación (Porras, 2005). Esta clasificación tiene la validez de emplear una variable objetiva, pero hay que advertir que el año de inauguración (término que consideramos más apropiado que el de creación) es tan solo una referencia que alude al momento en que se constituye la sociedad empresarial. Esta fecha no marca 
necesariamente el inicio de la producción del nuevo espacio esquiable (Canfranc, Candanchú, La Molina y otras estaciones actuales ya eran utilizadas por esquiadores antes de que existieran empresas concesionarias).

A este respecto, debe entenderse la creación de los espacios de nieve como un proceso que se dilata en el tiempo, sin que tan siquiera pueda considerarse concluido actualmente. Incluso la etapa de descubrimiento y aprovechamiento para la práctica del esquí puede ser anterior a la inauguración de la estación. El proceso de creación de los nuevos espacios resulta tan importante como el resultado final y las relaciones que se generan entre los diferentes elementos que lo conforman.

Por tanto, y una vez realizado el estudio de los diferentes espacios de nieve que existen en España, se ha intentado aplicar a estos los modelos conocidos de clasificación de estaciones de esquí, llegándose a las siguientes conclusiones: las tipologías establecidas por diferentes autores, tanto para el ámbito alpino como para España, sólo son de aplicación relativa y siempre mediante adaptación, en algunos casos forzada, al caso español. En este sentido, coincidimos en parte con Préau y su afirmación respecto a la dificultad de establecer una clasificación ajustada a todas las casuísticas.

Dicho esto, una propuesta tipológica de carácter territorial pasa por establecer una clasificación centrada principalmente en el espacio producido pero sin desechar el propio proceso de creación. En este sentido, entendemos que dos aspectos son fundamentales: la generación de espacios complejos de carácter polinuclear y polivalentes (aspecto que retomamos de Préau, 1968, y de Mesplier \& Bloc-Durafour, 2000), y de espacios más simples y siempre de condición aislada (que han considerado en sus estudios la casi totalidad de los autores consultados, como puede ser el caso del tercer tipo de estaciones de Préau o las estaciones de $2^{\mathrm{a}}$ y $3^{\mathrm{a}}$ generación de Lozato-Giotart, y los parques de nieve de los que hablan Cirici, 1973, Ganyet, 1985, en Porras, 2005, y López Palomeque, 1996).

Bajo estas premisas, podemos realizar la siguiente propuesta de clasificación:

1. Espacios polinucleares polivalentes. Estaciones de esquí asociadas a complejos turísticos. No necesariamente son los espacios más antiguos (estaciones de primera generación), pero sí parece existir una relación evidente entre "longevidad" y "complejidad" del destino. Son espacios maduros y consolidados, pero aún en desarrollo. El porcentaje de segundas residencias es altísimo, como cabría esperar (Gili, 2003), superando siempre el 90\%. Esto sucede salvo si existe un núcleo de mayor tradición turística, como La Molina, donde el porcentaje de segundas residencias disminuye ligeramente, situándose en 85,7\%.

Entrarían en esta categoría aquellas estaciones de esquí que comparten por proximidad dominios esquiables, es decir, un mismo recurso territorial y donde la construcción de la estación está vinculada al proceso inmobiliario del que depende en buena medida su viabilidad. Constituyen ejemplos de esta tipología las estaciones de La Molina-Masella, Candanchú-Astún, Baqueira-Beret, Navacerrada-Valdesquí y San Isidro-Fuentes de Invierno. Esta última es un caso singular, en tanto que se trata de una estación de desarrollo reciente (2007) e iniciativa pública9 cuya razón de ser obedece a una demarcación administrativa. En efecto, se aprovecha la existencia previa de la estación de San Isidro (provincia de León) para construir cerca, pero en otra comunidad autónoma (Asturias) las nuevas instalaciones. Podría decirse que el carácter polinuclear de este centro de esquí es circunstancial, debido a la situación limítrofe del recurso esquiable entre dos comunidades autónomas.

Este modelo de estaciones polinucleares y polivalentes obedece a una etapa avanzada del proceso de producción del espacio turístico de nieve. Coincide, normalmente y según se ha podido constatar, con los mejores emplazamientos, los más rentables en cuanto a días de nieve y están siempre vinculados con promociones urbanísticas. El caso paradigmático lo representa BaqueiraBeret, probablemente uno de los espacios más idóneos para el esquí (Gómez et al., 2016). Su evolución se produce en varias fases una vez se comprueba su viabilidad: Baqueira, en 1964, Beret, en 1984 y Bonaigua, en 1994. Constituye la única estación española considerada en la clasificación según su MPA como muy grande. Junto con Valdesquí, son las únicas de propiedad privada de cuantas se han considerado en este estudio.

2. Estaciones aisladas con urbanización turística. Espacios mononucleares y especializados donde se vincula el desarrollo de la estación de esquí al de la urbanización turística anexa o situada en la corona inmediata. Estaríamos ante un espacio que correspondería a un segundo nivel de complejidad, pues en ocasiones la urbanización de terrenos no se produce junto a la misma estación, lo que se traduce en un ámbito espacial más extenso. Muchos de los casos pertenecientes a la primera tipología han atravesado esta fase, por lo que podemos considerarla como un tipo de estadio de desarrollo. En este sentido, se podría hablar de espacios de una hipotética " 2 a generación" en el contexto del espacio de nieve español, que cronológicamente lleva un desfase significativo con respecto a los Alpes. Si se comprueba la fecha de inauguración de las estaciones encuadradas en esta tipología, se puede constatar una cierta relación entre el modelo estación-urbanización turística con la antigüedad del espacio, pues son todas estaciones construidas entre 1954 y 1970 (salvo Leitariegos, que es de 1986). Estas fechas se corresponderían con una etapa temprana del desarrollo del espacio de nieve en nuestro país.

Formarían parte de este grupo las siguientes estaciones: Valgrande-Pajares, Formigal, Alto Campoo, Sierra Nevada, La Pinilla, Cerler, Panticosa y Leitariegos, además de Port del Comte (no considerada, pues no forma parte de Atudem).

${ }^{9}$ Boletín Oficial del Principado de Asturias No 60 del lunes 14 de marzo de 2005. 
Habría que significar que ha sido complicado clasificar el caso de Formigal-Panticosa. Considerado de forma conjunta representaría un exponente de espacio polinuclear y polivalente (turismo especializado en Formigal, estación vinculada a pueblo tradicional de montaña y balneario de Panticosa, más la centralidad de Sallent de Gállego y su polivalencia en actividades deportivas de montaña y centro logístico y de gestión). Ambas estaciones están separadas por sólo $15 \mathrm{~km}$, sin embargo, entendemos que no comparten un mismo recurso territorial. Conforman realidades aisladas, aunque vinculadas por proximidad geográfica e incluso con gestión compartida centralizada en Sallent de Gállego, cuyo ayuntamiento es el principal promotor. Así interpretado, Formigal responde al modelo de estaciones de esquí vinculadas con promociones urbanísticas y lo mismo ocurre con Panticosa. Este último caso representa casi el único ejemplo de estaciónpueblo, dado que las infraestructuras están junto al núcleo, si bien es cierto que éste ha crecido como resultado de la existencia de la estación (456 habitantes en 1970 y 786 en 2018).

A este respecto, la inversión de la dinámica demográfica entre 1960 y 2018 según datos censales referidos a los municipios en los que se encuentran las estaciones parece evidenciar una correlación entre las estaciones de mayor tamaño (tipologías 1 y 2) y su influencia sobre la dinámica de la población. El efecto que sobre la población puede tener una estación de esquí es un tema que ya fue estudiado para el Pirineo por Lasanta et al. (2013). No obstante, para nuestro caso es aventurado afirmar con rotundidad la correlación de variables sin precisar el área de influencia y llevar a cabo un análisis más detallado.

3. Estación aislada más hotel o inmueble de apartamentos turísticos. Espacios mononucleares y especializados donde se vincula el desarrollo de la estación de esquí al de un inmueble para alojamiento turístico anexo. En realidad, responde a un modelo similar al anterior, si bien el resultado es más "compacto" y con una menor entidad territorial por resultar un espacio focalizado. Adoptar una forma $\mathrm{u}$ otra (urbanización turística o inmueble turístico en modalidad de hotel o apartamentos) creemos que es más una opción o modelo estratégico que una "fase o estadio", si bien en ambos casos parece que responden a etapas ya desarrolladas de espacios turísticos de nieve. En esta tipología se incluyen las estaciones de Val de Núria, Espot, Manzaneda (a caballo ya entre hotel y urbanización turística) y Port Ainé. El caso de Val de Núria es singular, pues aun tratándose de un espacio maduro que se inaugura en 1948 y que incluso cuenta con la implicación (e inversiones) muy potentes por parte de los agentes públicos, sin embargo no ha sido capaz sino de desarrollar un espacio mononuclear y muy acotado, debido principalmente a las condiciones de aislamiento del territorio circundante.

Estas estaciones son las que, a tenor del número medio de días abiertos por temporada (112 en los últimos 10 años), representan los espacios de nieve más desfavorecidos por las condiciones climáticas, con un índice que se sitúa por debajo de la media global de 116 días. No obs- tante, este parámetro no parece especialmente significativo, toda vez que los rangos varían entre los 112 y los 120 días, correspondiente este último a las estaciones del primer grupo.

4. Estación aislada. Es el espacio más simple, mononuclear y especializado, y consta únicamente de las infraestructuras propias de las estaciones de esquí, sin dotación de infraestructuras para alojamiento. En este grupo se encontraría la totalidad de estaciones de esquí nórdico o de fondo, previsiblemente porque desarrollan dinámicas territoriales más "tenues" que las estaciones de esquí alpino. Podría argumentarse que pueden equivaler en cierta medida a los parques de nieve de Cirici (1973), Ganyet (1985, en Porras, 2005) y López Palomeque (1996), pero esto sólo es cierto respecto al tipo de espacio construido y su funcionalidad, pero no en cuanto a su proximidad a los mercados. En este sentido, estos espacios están muy alejados de los principales focos emisores de demanda como para que se pueda considerar viable un uso diario de las estaciones, pues los viajes de ida y vuelta no bajan de las 4 horas. Por lo tanto, consideramos que más bien se trata de estaciones cuyo emplazamiento, no del todo adecuado, ha condicionado sus posteriores desarrollos. No obstante, sí que se trata en general de los espacios de nieve de construcción más reciente por lo que la variable antigüedad o longevidad también debe haber tenido repercusión sobre el resultado final del espacio producido.

Forman parte de esta tipología las estaciones de Valdelinares, Lunada, Vallter 2000, Valdezcaray, Boí Taüll, Tavascán, Javalambre, Sierra de Béjar-La Covatilla, y los espacios dedicados a esquí nórdico de Lles, Llanos del Hospital, Larra-Belagua y Puerto de la Ragua.

En el proceso de producción de estos espacios está presente de manera habitual la iniciativa pública, bien municipal, bien local, o frecuentemente ambas. Se eligen localizaciones no del todo adecuadas respecto a la demanda y su situación actual obedece tanto a un desarrollo temprano, como a una etapa abortada. Es el caso de Valdezcaray, iniciativa municipal que aportó el 50\% de la inversión inicial y que se basa en un modelo, copiado de Chamonix, donde no se apuesta por alojamientos en la estación para así propiciar el beneficio en la propia localidad de Ezcaray, promotora del proyecto.

En relación con la variable antigüedad o longevidad del espacio, lo que se ha dado en llamar estaciones de diferentes generaciones, en nuestra opinión este parámetro resulta de gran importancia, pero al comprobar su incidencia en los espacios españoles, concluimos que éste no es definitivo. No obstante, sí podemos afirmar que a mayor longevidad de un espacio, mayor es la tendencia a producir un destino polinuclear y polivalente y, por tanto, más complejo (casos de La Molina, Candanchú o Baqueira-Beret). No obstante, la variable de la antigüedad tiene un significado relativo que está relacionado con otros aspectos, como puede ser el aislamiento y la potencialidad del territorio, la implicación de los agentes públicos y privados, el nivel de inversión, la aceptación por parte de la demanda, la promoción y, en definitiva, aspectos más complejos que inciden sobre el proceso de 
producción de un destino de turismo de nieve, más allá de su antigüedad. Exponemos esto porque entendemos que puede ser un tema de interés para futuras investigaciones que podrían desarrollar este aspecto y completar e incluso corregir la propuesta tipológica que aquí se ha elaborado.

\section{Conclusiones}

El primer aspecto a destacar es que las casuísticas alpina y española son diferentes en cronología y características geográficas. En España, al comenzar la década de 1950, sólo existían cinco estaciones de esquí, por lo que prácticamente quedaría descartado el considerar estaciones de primera generación según el modelo y cronología alpinos. Además, debido a las condiciones climáticas, en España no se alcanza espesores de nieve aceptables hasta cotas superiores a los 1.500 metros. A semejante altitud ya no existen núcleos habitados o son muy escasos, por lo que los parámetros empleados para las clasificaciones de espacios alpinos, tales como altitud o vinculación con pueblos tradicionales de montaña, tienen un difícil encaje en el caso español.

Tras el análisis llevado a cabo se evidencia que:

1. La cronología española respecto a la producción de los espacios de nieve es más tardía que la alpina. En este último ámbito las estaciones de esquí se construyeron con antelación a las españolas y en un contexto geográfico e histórico distinto, circunstancia que afecta a la tipología de espacios producidos.

2. Aun considerando válido el criterio de clasificación de las estaciones por "generaciones", entendemos que éste no es aplicable al caso español. No se cuestiona su validez o no para el caso francés o alpino en general.

3. Sí parece existir una relación entre la antigüedad de las estaciones y la mayor o menor complejidad del espacio producido a su alrededor. A este respecto, quizás una variable interesante podría haber sido calcular las inversiones efectuadas en cada espacio, con el fin de analizar de qué manera influye en la mayor o menor complejidad del destino. Incluso podría estudiarse de manera pormenorizada la incidencia de una posible especulación con los terrenos y su urbanización. Pero esto no ha sido posible. Tampoco ha sido posible establecer un área de influencia de cada estación, cuestión que resultaría clave, pues no poder definir dicho ámbito dificulta el análisis espacial al no poder acotar de manera justificada hasta dónde ejerce una influencia territorial, económica o social una estación de esquí.

4. Tomando en consideración las diferentes variables que se han empleado en el presente estudio respecto de las dos coronas de análisis o ámbitos: inmediato $(0-5 \mathrm{~km})$ y próximo $(5-15 \mathrm{~km})$ y considerando principalmente el espacio producido, se estableció una propuesta clasificatoria que considera 4 tipos de espacios: estaciones de esquí asociadas a complejos turísticos, estaciones aisladas con urbanización turística, estación aislada más hotel o inmueble de apartamentos turísticos y estación aislada sin alojamientos en la corona inmediata. Dichos espacios se pueden clasificar según complejidad y especialización según el siguiente esquema:

1. Espacios complejos polinucleares y polivalentes. Estaciones de esquí asociadas a complejos turísticos.

2. Espacios simples mononucleares y especializados. Que se pueden resolver como

a. Estaciones aisladas con urbanización turística.

b. Estación aislada más hotel o inmueble de apartamentos turísticos.

c. Estación aislada sin alojamientos en la corona inmediata.

5. Por último, no todas las variables consideradas resultaron significativas para elaborar la propuesta final de clasificación tipológica. Las principales variables empleadas en esta clasificación han sido: el carácter aislado de la estación, la relación espacial con el sistema de poblamiento, la creación de espacios polinucleares o mononucleares, la especialización o polivalencia de los espacios, la antigüedad, la producción de viarios específicos de servicio y también, aunque en menor medida, las variables relacionadas con el tamaño, complejidad y especialización de la estación (número y capacidad de los remontes, momento de potencia, cañones de nieve, servicios ofertados, áreas y superficie de aparcamiento y oferta de alojamientos) y población municipal.

Por lo tanto y en síntesis, se descarta la posibilidad de establecer una clasificación por generaciones o períodos de entrada en funcionamiento de las estaciones de esquí. Creemos demostrado que existe una correlación entre la antigüedad de los espacios de turismo de nieve y la mayor o menor complejidad de los mismos, pero no es factible establecer una clasificación coherente según la longevidad, ni el tamaño o magnitud de las estaciones de esquí o la altitud de las mismas (criterio frecuente en el ámbito alpino). La articulación del espacio producido y las relaciones que se establecen entre sus componentes resultan más determinantes que el proceso de formación. Aún queda por diseñar un método que permita establecer cuál es el área de influencia de las estaciones de esquí, pues de su resolución es probable que derive una clasificación más completa desde el punto de vista geográfico y territorial, aspecto este que dejamos para futuras investigaciones.

\section{Referencias}

ATUDEM, 2018. El esquí español importa. https://drive.google.com/file/d/1LmfXNVKD6ursHkgkz8Tu5I07zZGYPP OI/view

Barker, M., 1982. Traditional Landscape and Mass Tourism in the Alps. Geographical Review, 72(4): 395-415. https://doi. org/10.2307/214593

Barrado, D. \& Calabuig, J., 2001. Geografia mundial del turismo. Editorial Síntesis, Madrid. 
Bayón, F. (Director), 1999. 50 años del turismo español. Un análisis histórico y estructural. Editorial Centro de Estudios Ramón Areces y Escuela Oficial de Turismo, Madrid.

Bourdeau, P., 2009. De l'après-ski à l'après-tourisme, une figure de transition pour les Alpes? Réflexions à partir du cas français. Revue de Géographie Alpine, 97 (3). https://doi. org/10.4000/rga.1049

Cardona, M. \& Dupre, Ll., 1985. Esports de neu a Catalunya. Centre excursionistas de Catalunya. Club Alpí Catalá. Editorial Montblanc, Barcelona.

Cirici, C., 1973. El esquí: juego de sociedad de los años 70. Cuadernos de Arquitectura y Urbanismo, 95: 2-6.

Fernández, L. Fernández, J. \& Fernández-Trapa, T., 1990. Esquí en los Pirineos. Historia para un futuro sin fronteras (II). EStudios Turísticos, 105: 79 - 99.

Galiana, L. \& Barrado, D., 2006. Los Centros de Interés Turístico Nacional y el despegue del turismo de masas. Investigaciones Geográficas, 39: 73-93. https://doi.org/10.14198/ INGEO2006.39.04

Gili, M., 2003. Las viviendas de segunda residencia. ¿Ocio o negocio? Scripta Nova. Revista Electrónica de Geografía y Ciencias Sociales, 7 (146). http://www.ub.es/geocrit/sn/sn146(052).htm

Gómez, M., Armesto, X. \& Cors, M., 2016. Crisis económica y estaciones de esquí en las comarcas catalanas de la Val d'Aran, Pallars Sobirà y Alta Ribagorça. Turismo y crisis, turismo colaborativo y ecoturismo. Societat d'Història Natural de les Balears, Palma de Mallorca.

Laguna, M. \& Nogués, D., 2002. La influencia del potencial del medio natural en el desarrollo turístico del Pirineo aragonés. XI Coloquio de Geografía Rural. Los espacios rurales entre el hoy y el mañana, 149 - 157 pp., Santander.

Lasanta, T., Laguna, M. \& Vicente-Serrano, S., 2007. Variabilidad espacial de los efectos socioeconómicos de las estaciones de esquí alpino en los municipios rurales del Pirineo Aragonés. Pirineos, 162: 109-176. https://doi.org/10.3989/ pirineos.2007.v162

Lasanta, T., 2010. El turismo de nieve como estrategia de desarrollo en el Pirineo aragonés. Cuadernos de Investigación Geográfica, 36(2): 143-161. http://dx.doi.org/10.18172/cig. vol36iss2

Lasanta, T., Beltran, O. \& Vaccaro, I., 2013. Socioeconomic and territorial impact of the ski industry in the Spanish Pyrenees: mountain development and leisure induced urbanization.
Pirineos, 168: 103-128. https://doi.org/10.3989/Pirineos. 2013.168006

Lasanta, T., Arnáez, J. \& Pascual, N., 2014. La contribución de una pequeña estación de esquí al desarrollo de su entorno: el caso de Valdezcaray (La Rioja). Cuadernos de Turismo, 33: 151-172.

López Palomeque, F., 1996. Turismo de invierno y estaciones de esquí en el Pirineo catalán. Investigaciones Geográficas, 15: 19-39. https://doi.org/10.14198/INGEO1996.15.04

Lozato-Giotart, J.-P., 1990. Geografía del turismo: del espacio contemplado al espacio consumido. Editorial Masson, Barcelona.

Martos, P., 2002. El sistema turístico-deportivo de las estaciones de esqui y montaña españolas, Universidad de Granada, Granada.

Mesplier, A. \& Bloc-Duraffour, P., 2000. Geografía del turismo en el mundo. Editorial Síntesis, Madrid.

OIU.,Oficina de Información Urbanística, 1973. El turismo de invierno y la planificación urbanística. Cuadernos de Arquitectura y Urbanismo, 95, pp. 12-19.

Pearce, D., 1989. Tourist development. Longman, Londres.

Pearce, D., 1999. Towards a geography of the geography of tourism: Issues and examples from New Zealand. Tourism Geographies, vol. 1. https://doi.org/10.1080/146166899087 21334

Perlik, M., 2011. Alpine gentrification: the mountain village as a metropolitan neighborhood. Revue de Géographie Alpine, 99(1). https://doi.org/10.4000/rga.1370

Porras, F., 2005. L'intervento pubblico sul turismo attivo. Il caso concreto delle stazioni sciistiche. Tesis doctoral (inédita). Universidad de Bolonia. Italia. Recuperada de http:// amsdottorato.unibo.it/124/

Préau P., 1968. Essai d'une typologie des stations de sports d'hiver dans les Alpes du Nord. Revue de géographie alpine, 56(1): 127-140. www.persee.fr/doc/rga_0035-1121_ 1968 num $56 \quad 13571$

Tritu, (2018). Clasificación de las Estaciones de Esqui Españolas y Portuguesa por Momento de Potencia. Temporada 2018/19. Recuperado de https://www.nevasport.com/rmeee/art/36267/ Clasificacion-de-las-Estaciones-de-Esqui-Espanolas-y-Portuguesa-por-Momento-de-Potencia-Temporada-201819/

Vanat, L., 2018. International Report on Snow \& Mountain Tourism. Recuperado de https://vanat.ch/international-reporton-snow-mountain-tourism.shtml 